\title{
COMPUTATION OF NONLINEAR ONE-TURN MAPS FROM MEASUREMENT WITH MODEL-INDEPENDENT ANALYSIS *
}

\author{
Chun-xi Wang, John Irwin, Yiton T. Yan \\ Stanford Linear Accelerator Center, ms 26, P.O. Box 4349, Stanford, CA 94309
}

\begin{abstract}
It is desirable to determine the nonlinear transformation maps, especially the one-turn map, of a storage ring from measured BPM data for nonlinear analysis in order to improve the machine performance. However, the accuracy of detecting the weak signals from nonlinear effects is often limited by the available BPM resolution. With the recent development of Model-Independent Analysis methods, which can significantly reduce BPM random noise via statistical analysis, it is possible to more accurately determine the nonlinear maps from measured data by using a large number of BPMs. Computational techniques and some simulation results for PEP-II will be presented.
\end{abstract}

\section{INTRODUCTION}

Although the map approach is very successful in single particle beam dynamics studies and dominates modern beam optics design tools, it is rarely used for beam diagnosis and control. In order to diagnose and improve beam dynamics in a ring, various techniques are used to measure the global properties such as chromaticity and tune-shift-withamplitude, which can also be extracted from a nonlinear one-turn map if measured. In reference [1] we argued the possibility to measure a nonlinear one-turn map with good accuracy, provided that the BPM resolution is sufficiently high. However, the required resolution is often not available.

Recently we developed Model-Independent Analysis (MIA) methods to study beam dynamics[2, 3]. One important achievement of MIA is the capability to significantly reduce random noise of individual BPM readings via statistical analysis of an ensemble of BPM readings of a large number of pulses at a large number of BPMs. Therefore MIA can facilitate/accomodate nonlinear map measurements. This paper will explore this possibility. We will first discuss how to apply MIA to nonlinear map measurements in general, then present some simulation results for the PEP-II high energy ring.

\section{MAP COEFFICIENTS AS PHYSICAL BASIS OF MIA}

The single particle beam dynamics can be represented by the transformation map $\mathcal{M}_{a \rightarrow b}$ that maps any initial phase space point $X^{a}$ at location $a$ to a phase space point $X^{b}$ at location $b$. Using a Taylor map representation, $\mathcal{M}_{a \rightarrow b}$ may

\footnotetext{
* Work supported by the Department of Energy, contract DE-AC03$76 \mathrm{SF} 00515$.
}

be written as

$$
\begin{aligned}
X_{k}^{b}= & C_{k}^{b}+R_{k l}^{a \rightarrow b} X_{l}^{a}+T_{k l m}^{a \rightarrow b} X_{l}^{a} X_{m}^{a}+ \\
& U_{k l m p}^{a \rightarrow b} X_{l}^{a} X_{m}^{a} X_{p}^{a}+V_{k l m p q}^{a \rightarrow b} X_{l}^{a} X_{m}^{a} X_{p}^{a} X_{q}^{a}+\cdots
\end{aligned}
$$

where the summation convention on the repeated indices is assumed. $X_{k}$ is the $k$-th component of a phase space vector. $C_{k}^{b}$ is the zero-order term of the $k$-th component and should vanish if the closed orbit is chosen as a reference. $R^{a \rightarrow b}$, $T^{a \rightarrow b}, U^{a \rightarrow b}$, and $V^{a \rightarrow b}$ are the usual TRANSPORT notations for the 1st, 2nd, 3rd, and 4th order map coefficients.

Now consider BPM readings for $P$ pulses/turns at $M$ locations $b_{1}, b_{2}, \cdots, b_{M}$ in a ring, obviously we can cast the BPM-reading matrix $B$ for the horizontal plane into a physical base decomposition via Eq.(1),[2, 3]

$$
B=Q F^{T}
$$

where $F$ consists of all coefficients in the $x$ components of the maps as the physical basis (see Fig.2) and $Q$ contains the corresponding initial conditions, such as

$$
F=\left[\begin{array}{cccccc}
C_{1}^{b_{1}} & R_{11}^{a \rightarrow b_{1}} & R_{12}^{a \rightarrow b_{1}} & \ldots & T_{111}^{a \rightarrow b_{1}} & \ldots \\
C_{1}^{b_{2}} & R_{11}^{a \rightarrow b_{2}} & R_{12}^{a \rightarrow b_{2}} & \ldots & T_{111}^{a \rightarrow b_{2}} & \ldots \\
\vdots & \vdots & \vdots & & \vdots & \\
C_{1}^{b_{M}} & R_{11}^{a \rightarrow b_{M}} & R_{12}^{a \rightarrow b_{M}} & \ldots & T_{111}^{a \rightarrow b_{M}} & \ldots
\end{array}\right]
$$

and

$$
Q=\left[\begin{array}{cccccc}
1 & x_{1}^{a} & p_{x_{1}}^{a} & \cdots & \left(x_{1}^{a}\right)^{2} & \cdots \\
1 & x_{2}^{a} & p_{x_{2}}^{a} & \cdots & \left(x_{2}^{a}\right)^{2} & \cdots \\
\vdots & \vdots & \vdots & & \vdots & \\
1 & x_{P}^{a} & p_{x_{P}}^{a} & \cdots & \left(x_{P}^{a}\right)^{2} & \cdots
\end{array}\right]
$$

Now the constant terms $C_{1}^{b}$ 's may contain BPM offsets also. The best way to take out such terms is to use the measured closed orbit as the reference and use the difference orbits to construct $B$. It is better not to use the average orbit as the reference because the high order terms may not average to zero and yield significant errors, when the beam is excited to large amplitudes, which is necessary in order to measure the high order map coefficients. On the other hand, it is possible to get a very accurate closed orbit in a ring by averaging over a large number of turns of the unperturbed beam.

Similarly one can construct a physical base decomposition for the vertical BPM readings with the $y$ components of the maps. However, to get the $x^{\prime}$ and $y^{\prime}$ components of the maps, such information at each BPM is required. Note that the $Q$ matrix is the same for all components. In 
fact, one can stack the BPM readings for both planes into one matrix and extend the physical base decomposition to cover both planes. However, the benefits of doing so are still not clear.

One particularly interesting nonlinear map is the oneturn map of a ring. To accommodate this into Eq.(2), $F$ should contain the one-turn map coefficients and $B$ should contain the measured phase-space variables one-turn after the initial values used in $Q$. Reference [1] has a concrete example. Note that in this case, $F$ does not contain any BPM patterns as in the usual MIA application. However, the $Q$ matrix is still the same.

Standard least-squares fitting can be used to solve Eq.(2) for the map coefficients. The difficulty is to get a sufficiently accurate $B$ and $Q$.

\section{PHASE-SPACE MEASUREMENT AND SVD NOISE REDUCTION}

To measure the weak nonlinearity in phase-space dynamics of a ring, the background has to be sufficiently clean. MIA provides a nice way to check this requirement. First obtain a BPM-reading matrix $B_{P \times M}$ by recording a large number (e.g. $P=5000$ ) of turn-by-turn data at all available BPMs (e.g. $M=150$ ) with the stored beam unperturbed. Then check the singular value spectrum of $B$. Ideally it should contain only the BPM noise floor since all physical motions should be well damped. Very noisy BPMs can be easily identified at this stage. Any other significant modes indicate systematic BPM errors and/or physical sources exciting the beam. Such problems need to be fixed in order to pursue nonlinear map measurements. We assume the singular value spectrum is clean. We will take the average orbit as the reference orbit and identify the noise level for later use.

Now measure another BPM-reading matrix with large (e.g. $8 \sigma$ ) betatron oscillations excited by fast kickers for example. Subtract the mentioned reference orbit from each measured orbit in order to get rid of BPM offsets and define the expansion points of the measured maps. At this stage, two MIA procedures can be employed to improve the phase-space dynamics measurement: SVD noise reduction and degrees-of-freedom analysis.

To reduce the random noise, compute a Singular Value Decomposition (SVD) as $B=U S V^{T}$, identify the noise floor or use the noise level mentioned above, set the corresponding noise singular values to zeroes, and then remultiply these matrices to construct a noise-cut matrix $\underline{B}$. This simple procedure can reduce the BPM random noise by a factor of $\sqrt{\frac{d}{M}}$, where $d$ is the number of remaining singular values above the noise floor. Depending on the situation, such noise reduction could be rather significant, especially when the BPM resolution is poor and one is struggling to measure a few leading nonlinear coefficients.

The degrees-of-freedom analysis could help to locate the best linear section in the ring for phase-space variable mea- surements. Then two BPMs in each plane are used to determine the initial conditions using the noise-cut data. In principle, one can use the orthogonal linear modes as the phase-space variables. However, it is probably better to use the model of the mentioned linear section to define the phase-space variables from orbit measurements. Note that even if the linear machine model might not be sufficiently accurate, it will not affect the sensitivity of nonlinear map measurement.

\section{SIMULATION FOR PEP-II}

Simulations for PEP-II high energy ring have been carried out to investigate the feasibility of nonlinear map measurements. 5000 turn data at all BPMs (147 for each plane) were generated by tracking 200 turns of 25 randomly chosen $x$ and $y$ initial conditions (no energy change) within $10 \sigma$ ranges. In addition, various levels of random noise were added in order to test the sensitivity of map measurements and the effects of noise reduction described in section 3. BPM resolutions are randomly selected from the specified ranges.

Fig. 1 shows the singular value spectra of the simulated system. Linear coupling and nonlinear modes are orders of magnitude weaker than the two dominant betatron modes - the reason nonlinear map measurements is challenging. The main frame shows the tail part of the $x$ spectra in detail. Note that the noise floor is about $10 \mu \mathrm{m}$, much lower than the individual BPM resolutions - a statistical benefit. Otherwise, all the nonlinear signals would be below the $100 \mu \mathrm{m}$ noise level. The arrow indicates where to cut off the noise floor.

Table 1 shows the rms errors of dynamical variable measurements for various BPM resolutions and the effects of noise-cut. Despite noticeable fluctuations, the accuracy of phase-space measurements is significantly improved, which makes nonlinear map measurements feasible with-

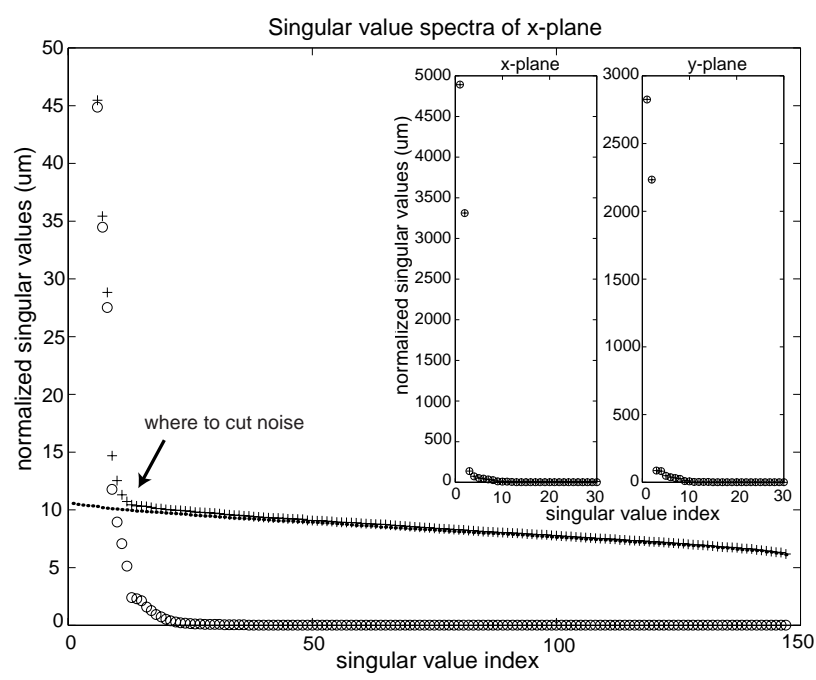

Figure 1: Singular value spectra of simulated data with "o" for signal only, "." for $100 \pm 20 \mu \mathrm{m}$ BPM noise only, and "+" for both. Insertions show the full vertical scale. 
Table 1: rms errors of phase-space variable measurements

\begin{tabular}{|c||c|c|c|c||c|c|c|c|}
\hline \multicolumn{1}{|c||}{$\begin{array}{c}\text { BPM reso- } \\
\text { lutions }(\mu m)\end{array}$} & \multicolumn{3}{c||}{ without noise-cut } & \multicolumn{3}{c|}{ with noise-cut } \\
\cline { 2 - 9 } & $x(\mu m)$ & $x^{\prime}(\mu r)$ & $y$ & $y^{\prime}$ & $x$ & $x^{\prime}$ & $y$ & $y^{\prime}$ \\
\hline $100 \pm 20$ & 102 & 7.1 & 156 & 14. & 36. & 2.6 & 25. & 2.1 \\
\hline $80 \pm 16$ & 74. & 6.4 & 126 & 11. & 17. & 2.1 & 24. & 2.0 \\
\hline $60 \pm 12$ & 55. & 4.9 & 103 & 9.3 & 12. & 2.4 & 20. & 1.8 \\
\hline $40 \pm 8$ & 30. & 2.3 & 56. & 5.1 & 8.2 & 1.2 & 14. & 1.3 \\
\hline $20 \pm 4$ & 20. & 1.7 & 30. & 3.4 & 10. & 1.1 & 11. & 1.3 \\
\hline
\end{tabular}

out stringent BPM resolution requirements.

Fig. 2 plots the linear and a few nonlinear map coefficients along all the BPMs. They are normalized to the $10 \sigma$ of phase-space variables, thus reflect the strength of each nonlinear term near the border of dynamical aperture. The apparent non-sinusoidal patterns in $R_{11}$ and $R_{12}$ are due to the uneven BPM locations. The peaks in the 2 nd order coefficients are due to the main sextupoles around the interaction point at the center. Such spatial patterns form the physical bases for the BPM readings. Simulation re-

$10 \sigma$ normalized map (x-component) coefficients
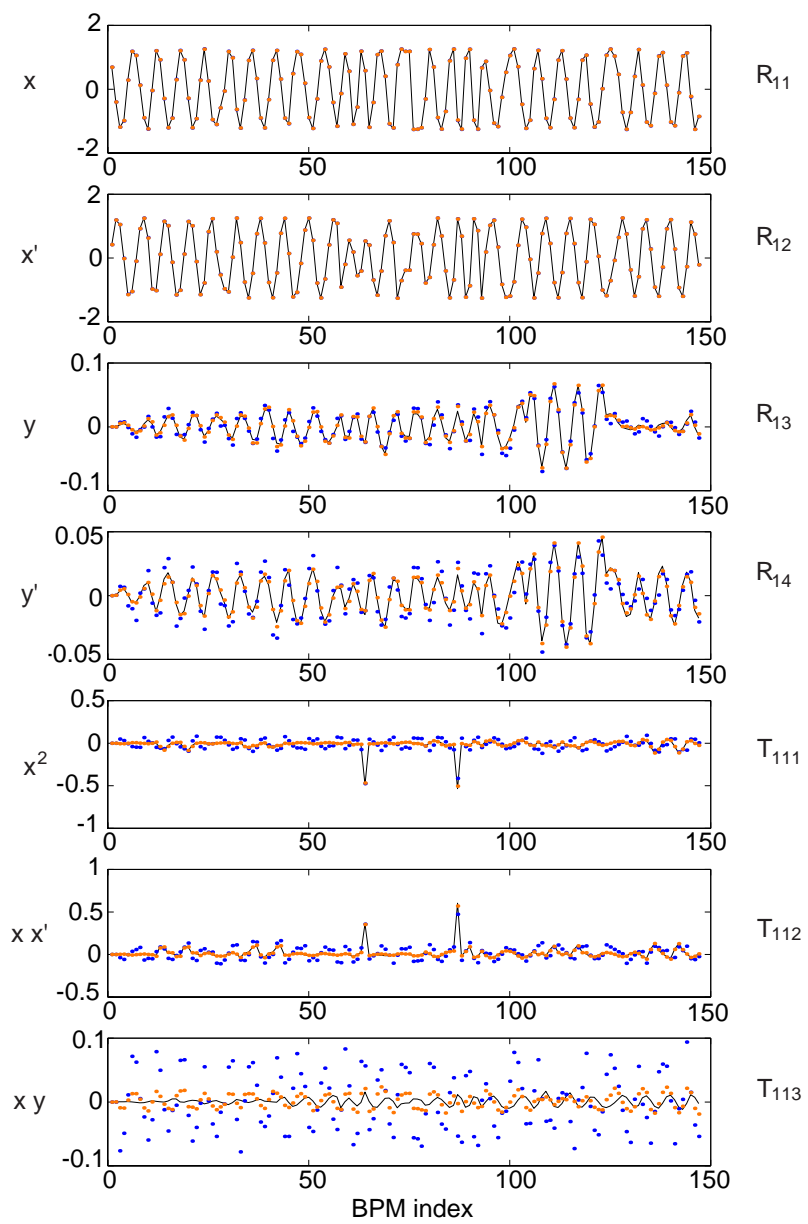

Figure 2: Map coefficients as physical basis. Only a few terms are shown. Solid lines are the exact values and dots are the simulated measurements. The lighter/darker dots are with/without noise-cut. (The on-line version shows the dots in different colors.)
Figure 3: $10 \sigma$ normalized one-turn map coefficients up to the 3rd order (x-component). "०" and "•" are for $100 \pm$ $20 \mu \mathrm{m}$ BPM noise, with and without noise-cut respectively.

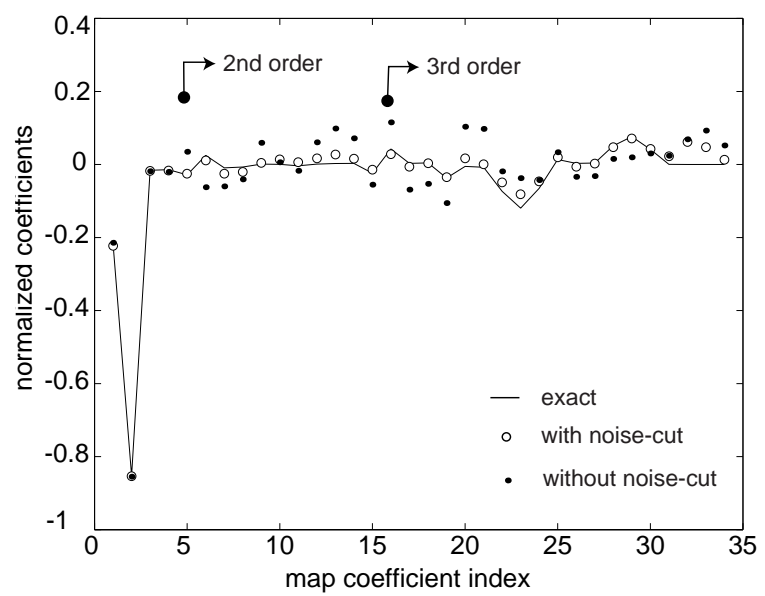

sults are also shown for the case of $100 \mu \mathrm{m}$ BPM resolutions, with and without noise-cut, on top of the exact solid curves. Although the main features can be obtained even without noise-cut, the accuracy is significantly improved by the noise-cut, which is crucial (after all, everyone knows where the sextupoles are). Note that the linear coupling terms $R_{13}$ and $R_{14}$ can be obtained rather accurately. Such information can be used to calculate the global linear coupling coefficient and furthermore help to localize the coupling sources. The errors in $T_{113}$ are due to the weakness of this nonlinear coupling term.

Fig. 3 plots the results of simulated one-turn map measurement with $100 \mu \mathrm{m}$ BPM resolution. Again the effect of the noise-cut is obvious. The accuracy is sufficient to reveal useful nonlinearity information. For example, a few percent error of the main sextupole strength should be detectable according to the simulations. More effective methods are under investigation.

\section{CONCLUSION}

Measurements of nonlinear (especially low order) maps in a ring become feasible with MIA methods, provided that systematic BPM errors are tolerable. Nonlinear map measurements can yield localized as well as global (one-turn map) nonlinearity information in a ring, which is valuable to beam dynamics study and machine improvement.

\section{REFERENCES}

[1] Chun-xi Wang and John Irwin, "Possibility to measure the Poincare section map of a circular accelerator", SLAC-PUB7547

[2] J. Irwin, C.X. Wang, Y.T.Yan, K.L.F. Bane, Y. Cai, F.-J. Decker, M.G. Minty, G.V. Stupakov, and F. Zimmermann, Phys. Rev. Lett. vol.82 no.8 p.1684 (1999)

[3] Chun-xi Wang, John Irwin, Karl Bane, Yunhai Cai, Michiko Minty, Franz J. Decker, Yiton T. Yan,"Model-Independent Analysis of beam dynamics in accelerators", to be published. 